\title{
La investigación escolar
}

\author{
Oscar Yecid Aparicio Gómez ${ }^{1}$
}

Recibido: 30-07-2018

Aceptado: 28-09-2018

El aprendizaje colaborativo y la construcción del conocimiento nos conducen a un ámbito superior que desemboca en el pensamiento crítico y el planteamiento de problemas significativos para la comunidad educativa. Estamos proponiendo un acercamiento al papel de la comunidad educativa, y específicamente de los estudiantes como comunidad de investigación escolar. Piaget resalta la auténtica actividad investigativa precisamente en la esfera de la reflexión, la abstracción y el lenguaje:

"Finalmente, se ha entendido que una escuela activa no es una escuela de labor manual. La actividad del niño en algunos niveles necesariamente conlleva la manipulación de objetos y aún una cierta agrupación física real, debido a que las nociones lógico-matemáticas, por ejemplo, se derivan, no de los objetos manipulados, sino de las acciones del niño y su coordinación. En otros niveles la más auténtica actividad investigativa tiene lugar en la esfera de la reflexión, de la más avanzada abstracción, y de la manipulación verbal a condición de que sean espontáneas y no impuestas al niño por el riesgo de permanecer parcialmente incomprendidas." (Piaget, 1980: 81)

La opción por una educación escolar integral que considere la importancia de la investigación en el aula a través de prácticas innovadoras depende también de actores externos a la relación directa entre el profesor y los estudiantes en el aula (Pérez, 2013). Por tanto, la organización de la sala de clases y las características personales de los profesores y estudiantes, así como las tareas y su contextualización,

1. Doctor en Filosofía (2006) y Doctor en Educación (2015) por la Universidad de Barcelona. Profesor y Editor. Universidad Nacional Abierta y a Distancia (UNAD), Colombia. Bogotá - Colombia.

Correo: oscar.yecid@gmail.com

ORCID: https://orcid.org/0000-0003-3535-6288 
interés y significatividad, deben sentirse apoyadas por la organización del establecimiento escolar y características personales de los administradores y líderes de la comunidad, y en última instancia por las políticas nacionales y regulaciones internacionales (Ferreyra, 2014).

Tonucci (1982) afirma que si se pretende hacer un cambio hacia una escuela constructiva, se requieren no solo acciones específicas sino nuevos presupuestos globales de la educación. Debería pensarse que los educandos saben que vienen a la escuela a compartir y a aumentar sus conocimientos precedentes, y que el maestro es una garantía del mayor aprovechamiento de la contribución de todos y que la inteligencia se modifica y articula con la estructuración de nuevos conocimientos. Para que estos presupuestos puedan lograrse es necesaria la activa participación de los estudiantes y el uso de un método de la investigación donde se aborden problemas que faciliten una motivación intrínseca (Tahull, 2016). Esto también puede determinarse desde el reconocimiento de lo próximo para reforzar la confianza en lo conocido y en los compañeros como base de nuevos conocimientos, para despertar la seducción por el conocimiento y la exploración (Langer, 2016).

La escuela debe facilitar espacios de encuentro y de confrontación de opiniones como fuente de investigación, privilegiando la inventiva y la creatividad del estudiante y su lugar determinante entre la investigación y la enseñanza. Se requiere un ambiente educativo que se adapte a la confrontación, y se adapte a la exploración creativa para el trabajo en equipo (de la Calle et al., 2014). Los tipos de investigación deberían originarse desde la memoria colectiva, donde incluso las paredes contengan dibujos y expresiones memorísticas del grupo, facilitando el lenguaje como el principal filtro de la experiencia (Tonucci, 1986).

Siguiendo a Tonucci (1986:16), aceptamos que la lección es "el instrumento coherente óptimo de transmisión de conocimiento", en la escuela transmisiva, pero que si apostamos por una escuela constructiva, o "construcActiva", el instrumento coherente óptimo es la investigación, a la que "le damos el mismo sentido que le da el científico y el investigador, con la modificación de los objetivos y de los instrumentos, de manera que sean adaptados a las virtualidades y exigencias de los niños, sin disminuir su coherencia y corrección." (Tonucci, 1986:17).

Lissón (1989) concreta la propuesta de investigación en el aula de Tonucci (1979 y 1986) en el siguiente esquema que hemos adaptado: 
- Planificación (Tema y límites del tema): ¿Quién lo hace? ¿Qué se sabe o sabemos del tema? ¿Qué quiero o queremos saber?

- Ejecución

- Método o procedimiento (guion de investigación): Recogida de datos, Observación, Descripción de las partes, Lectura de documentos y/o libros, Realización de encuestas y/o esquemas y/o gráficos.

- Elaboración e interpretación de datos: Trabajo "en la carpeta" (apuntes, subrayado, resumen, ilustraciones, construcción de materiales).

- Conclusiones: Delimitación de las conclusiones, Cosas que quedan por saber y porqué, Maquetación, Presentación escrita y/o oral.

- Control: Comunicación y Evaluación

Esta propuesta de planificación de la investigación, está muy conectada con los enfoques de alfabetización informacional y más concretamente con las competencias informacionales, las cuáles podemos definir como:

"como un conjunto de competencias asociadas con el acceso (búsqueda, localización, recuperación...), el procesamiento (análisis, interpretación, valoración, selección, reelaboración, representación...) y el uso (comunicación, aplicación, expresión, compartición...) de cualquier tipo y morfología de información (textual, auditiva o visual, así como hipertextual, y sus combinaciones en audiovisuales y multimedia), con independencia de su soporte (papel, magnético, óptico o digital) y de su tangibilidad." (Punt de Lectura, 2014).

En la siguiente tabla comparativa aparecen las fases de la investigación escolar que propone Tonucci, relacionadas con los estándares del desarrollo de habilidades informativas para el aprendizaje permanente de la International Federation of Library Associations and Institutions - IFLA (Lau, 2007), el modelo Modelo Gavilán de Competencias de Manejo de la Información (Eduteka, 2007), el modelo PAPUS de Preparación, Acceso, Procesamiento y Uso (Quintana, 2014a y 2014b), y el modelo 3.3.3 de Durban y Blasco (2012). 
Tabla 1. Comparativo de fases de la investigación escolar y competencias informacionales.

\begin{tabular}{|c|c|c|c|c|}
\hline Tonucci & IFLA & Gavilán & PAPUS & Modelo $3 \cdot 3 \bullet 3$ \\
\hline Planificación & & $\begin{array}{l}\text { Definir el } \\
\text { problema de } \\
\text { información y } \\
\text { qué se necesita } \\
\text { indagar para } \\
\text { resolverlo }\end{array}$ & $\begin{array}{l}\text { Preparación } \\
\text { (Planificación) }\end{array}$ & \\
\hline $\begin{array}{c}\text { Ejecución } \\
\text { (Método o } \\
\text { procedimiento) }\end{array}$ & $\begin{array}{c}\text { Acceso } \\
\text { (Definición y } \\
\text { articulación de } \\
\text { la necesidad } \\
\text { informativa, } \\
\text { Localización de la } \\
\text { información) }\end{array}$ & $\begin{array}{l}\text { Buscar y evaluar } \\
\text { fuentes de } \\
\text { información }\end{array}$ & $\begin{array}{l}\text { Acceso a la } \\
\text { información } \\
\text { (Búsqueda, } \\
\text { Localización, } \\
\text { Recuperación...) }\end{array}$ & $\begin{array}{l}\text { Buscar la } \\
\text { información }\end{array}$ \\
\hline $\begin{array}{l}\text { (Elaboración e } \\
\text { interpretación de } \\
\text { datos) }\end{array}$ & $\begin{array}{l}\text { Evaluación } \\
\text { (Evaluación de } \\
\text { la información y } \\
\text { Organización de } \\
\text { la información) }\end{array}$ & $\begin{array}{c}\text { Analizar la } \\
\text { información }\end{array}$ & $\begin{array}{c}\text { Procesamiento } \\
\text { de la información } \\
\text { (Ordenar, } \\
\text { Clasificar, } \\
\text { Organizar..., } \\
\text { Redactar, } \\
\text { Reescribir, } \\
\text { Refundir..., } \\
\text { Dibujar, Graficar, } \\
\text { Representar..., } \\
\text { Reelaborar, } \\
\text { Interpretar...) }\end{array}$ & $\begin{array}{c}\text { Tratar la } \\
\text { Información }\end{array}$ \\
\hline $\begin{array}{c}\text { Conclusiones } \\
\text { Control }\end{array}$ & $\begin{array}{l}\text { Uso (Uso de la } \\
\text { Información y } \\
\text { Comunicación y } \\
\text { uso ético de la } \\
\text { información) }\end{array}$ & $\begin{array}{l}\text { Sintetizar la } \\
\text { información y } \\
\text { utilizarla }\end{array}$ & $\begin{array}{c}\text { Uso de la } \\
\text { información } \\
\text { (Comunicar, } \\
\text { Aplicar, Expresar, } \\
\text { Crear, Compartir, } \\
\text { Difundir...) }\end{array}$ & $\begin{array}{l}\text { Comunicar } \\
\text { conocimiento }\end{array}$ \\
\hline
\end{tabular}

Fuente. Elaboración propia.

En la tabla anterior puede observarse la relación existente entre las fases de investigación en el aula que sostienen nuestro proyecto, y las fases de las competencias informacionales, según distintos autores, que evidencia la relación existente entre ellos, ya que ambos enfoques se basan en trabajo con información.

\section{La Investigación en la Institución Educativa}

La ampliación del currículo en las instituciones educativas que se preocupan por el aprendizaje colaborativo y la construcción del conocimiento deben optar por la construcción de un marco común para la investigación (Gordon, 2003). Esta condición básica permitirá que los estudiantes adquieran cierta profundidad de comprensión. En vez de 
resultados, se enfatizará en el proceso, así como en la importancia de la investigación, la construcción del conocimiento y la colaboración entre pares (Viejo, Cabezas y Martínez, 2013).

Onrubia, Colomina, y Engel (2008) proponen que los profesores y estudiantes deberían trabajar de manera similar a la de una comunidad científica colaborativa, planteando problemas y definiendo objetivos, aportando, intercambiando y contrastando ideas e información, colaborando unos con otros y construyendo progresivamente una base de conocimientos compartida.

Esta concepción de la comunidad educativa no solo aplica a los entornos académicos, sino que se orientan a la consolidación de ambientes específicos para la investigación en la Escuela, donde profesores y estudiantes puedan converger en temas de interés y ser acompañados unos y otros en el desarrollo de objetivos comunes (Barrantes, Beltrán \& Pérez, 2016). Una comunidad en la que se formulen preguntas y puedan descubrirse posibilidades reales para su resolución es donde se aprende a través de la actividad colaborativa, significativa y orientada a un fin.

En las aulas de clase puede reconocerse un cambio de actitud en los estudiantes y profesores cuando se encuentran cuestiones que verdaderamente quieren responder entre todos. La promoción de la creatividad y la innovación que puede producirse en los entornos educativos puede verse amplificada en comunidades de investigación. La innovación puede ser sostenida en el tiempo, ser aplicada a distintos contextos y replicada en ambientes diversos (Konieczny, 2015).

\section{La práctica investigativa}

Popper (1957: 87) afirma que: "Todas las teorías son experimentos, hipótesis provisionales, puestas a prueba para observar si funcionan; y toda demostración experimental es sencillamente el resultado de las pruebas llevadas a cabo con mi espíritu crítico, en un intento de averiguar dónde yerran nuestras teorías". La acción educativa es el preámbulo de la acción investigativa. En la medida en que una se orienta a la otra y luego la complementa puede verse proyectada en sus motivaciones iniciales. La persona que aprende debe sentirse acompañada en la búsqueda de significados y en la comprensión de las situaciones que se construyen en 
el tiempo, mientras se revisan, evalúan y perfeccionan los proyectos al interior de cualquier comunidad humana (Pérez, 2014).

Gardner (1983) y Wells (1999) destacan que hacer énfasis en el conocer más que en el conocimiento tiene también la ventaja de llamar la atención sobre los diferentes modos de conocer que intervienen en la resolución de la gran cantidad de problemas que encontramos en la vida diaria. El acto de conocer es fuente de conocimiento. Memorizar conocimientos previos únicamente, frenaría las posibilidades de creatividad que trae consigo el deseo conocer de manera permanente en la vida.

De hecho, la curiosidad es el motor del acto de conocer. Cuando la curiosidad sobre una situación es compartida se impulsan las comunidades de investigación que no se limitan a resolver problemas, sino que se comprometen en un proceso de descubrimiento que constituye su propia recompensa (Bennis y Biederman, 1997:17). Por tanto, hacer preguntas difíciles, recoger datos válidos, analizarlos minuciosamente y comprobar consistentemente las inferencias extraídas generan comunidades de investigación cuyos estudios y resultados se hacen significativos siempre que tengan consecuencias para la acción fuera del aula (Wells, 1997).

Los problemas bien estructurados presentan todos los elementos del problema; requieren un número limitado de reglas y principios que se organizan en una disposición previsible y prescriptible; poseen respuestas correctas y convergentes, y tienen un proceso de solución presentado y prescrito. Los problemas mal estructurados tienen unos objetivos vagamente definidos o poco claros, y restricciones no expuestas; tienen muchas soluciones, vías de solución o ninguna solución y requieren que los estudiantes expresen su opinión personal o sus creencias sobre el problema, así que hablamos únicamente de actividades humanas interpersonales (Jonassen, 1997: 68).

La formulación de los problemas de investigación necesita una metodología prevista y consensuada por la comunidad interesada. Esta base que soporta el camino hacia los aportes a la ciencia y al conocimiento en general necesita de la argumentación como variable que predice el éxito del estudiante ante problemas bien y mal estructurados (Hong, Jonassen, y Mcgee, 2003). Para evaluar el progreso alcanzado por la comunidad de investigación y por cada uno de sus miembros, es fundamental 
aplicar esta evaluación hacia la capacidad del estudiante para utilizar sus conocimientos y habilidades para resolver algún problema significativo y de su interés así como una valoración de las estrategias utilizadas en ese proceso.

El profesor ayuda a los estudiantes a generar preguntas que guíen la búsqueda, y por tanto: "Debe propiciar y ofrecer las pautas y guías para que construya y elabore por sí mismo o en colaboración con los otros, el conocimiento que debe adquirir, que cuestione las ideas o conceptos que se le ofrecen, que compare las teorías y/o modelos antagónicos (Area y Adell, 2009: 11).

Para ello Monereo y Fuentes (2008: 404) afirman que "Se debe ayudar al estudiante a reflexionar conscientemente sobre las estrategias de búsqueda empleadas y promover su conocimiento condicional o estratégico sobre cuándo y por qué es preferible emplear uno u otro procedimiento de búsqueda. [...] Se trata de enseñar a los estudiantes a documentar convenientemente la información localizada y utilizada según las normativas de citación más conocidas".

Newman (1987) nos dice que, en cuanto profesores, debemos estar dispuestos a sorprendernos; también debemos estar dispuestos a investigar la discordancia entre nuestros supuestos básicos y lo que ocurra en realidad que dé lugar a la sorpresa. El profesor debe ser también un investigador que modela las disposiciones y las acciones del aprendizaje a través de la pregunta y también dirige sus propias preguntas aspirando a mejorar la calidad y eficacia de la actividad de la comunidad (Gordon, 2003: 14).

\section{Aprender investigando}

Cuando los estudiantes aprenden a través de la acción, la reflexión y la demostración, centramos la atención en plantear problemas así como en resolverlos mediante el procedimiento de la investigación (Short y Burke: 1991). Al tratar de responder sus preguntas, los estudiantes trabajan para alcanzar unos objetivos que tienen sentido para ellos $\mathrm{y}$, a menudo, para una comunidad mayor, que puede ser el aula, la escuela o la comunidad externa. Descubren que existen vías alternativas de investigación de un problema y que, llegados a un aspecto determinado, si es posible 
buscar respuestas a muchas cuestiones diferentes y, muy importantes, descubren que no tiene por qué haber una solución o respuesta a un problema determinado (Rosebery, Warren y Conant, 1992: 63).

Chambers (1985) concluyó que los niños son críticos naturales, pero nosotros los desconocemos porque no escuchamos ni hacemos las preguntas correctas. Vygotsky (1987) considera que el aprendiz tiene más éxito en colaboración que si está solo. Wells (1999) señala que la comprensión se consigue cuando los aprendices tienen la oportunidad de transformar la experiencia y la información mediante actividades de construcción del conocimiento, en particular mediante oportunidades para un discurso progresivo (Brooks y Donato, 1994). Por tanto, al procurar que las ideas resulten significativas para otros, y a través del diálogo y el discurso los estudiantes pueden tener la sensación de lograr una mejor más clara comprensión.

Los niños aprenden a desarrollar y verificar sus propias ideas, equivocarse sin frustración, a evaluar los errores de modo tal que les permite acercarse paso a paso a la meta propuesta. Observan de inmediato las contradicciones entre lo que intentan hacer y lo que realmente sucede, y el error se convierte en una fuente de comprensión, ya sea para un proceso de aprendizaje, ya sea para la resolución de un problema (Papert, 1982: 8).

Para motivar la auténtica investigación, el estudiante debe recoger y "hacer suya" la pregunta (Van Tassell, 1994). Debe tenerse en cuenta que el objetivo de la investigación sea hacer, no aprender, porque en el hacer ya está implícito el aprender, y la motivación de los estudiantes se dirige habitualmente a lo práctico, al hacer de cada día. En este sentido, Brooks y Donato (1994) destacan que los estudiantes reinterpretan los objetivos del profesor a medida que desarrollan las actividades. Motivados y retados por preguntas y problemas reales, su atención se dirige a crear respuestas y soluciones.

Pozuelos y Travé (2005) afirman que el aprendizaje por investigación en el marco de la enseñanza se constituye como un mecanismo para dejar de lado la transmisión de conocimiento, superando así el concepto de "argumento de autoridad", para dar un lugar preponderante a la investigación como forma de apropiación del conocimiento por parte de los mismos estudiantes. Sobre las modalidades de investigación proponen: 
- La aplicación del método científico o experimental. Parte de situar al estudiante en una posición de científico con una situación problemática.

- El aprendizaje por descubrimiento. Presenta una situación que se aborda desde el tanteo experimental como medio de descubrimiento autónomo, de esta forma, el estudiante permanece altamente motivado y le impulsa a abordar nuevas investigaciones.

- La investigación guiada. Parte de problemas y orientaciones dadas por el docente donde los estudiantes realizan actividades a partir de un cronograma de actividades.

- La investigación del medio. Tiene como principio básico la problematización de la realidad motivando al análisis crítico y fundamentado, conectando a los estudiantes con sus inquietudes e intereses.

- Investigar problemas sociales de interés. Experiencias de investigación donde se abordan problemáticas que afectan el interés común.

- Investigación escolar y cambio conceptual. Concibe el abordaje desde el cambio en la investigación escolar como estrategia de enseñanza pero diferenciando la etapa educativa: primaria, secundaria, superior.

- Los proyectos de trabajo e investigación. Se desprende de una serie de cuestiones o inquietudes con índices y red de preguntas para abordar los proyectos.

- Las Webquest. Corresponde a la enseñanza por investigación en la era digital y dado su uso se le define como "investigación escolar en la red" en la cual se organizan los estudiantes por equipos de trabajo colaborativos a los cuales se les propone un problema que termina en un producto desarrollado con herramientas y documentación de Internet. Se concibe aquí un enfoque colaborativo y una dinámica de amplia indagación sobre un modelo flexible. 
Podemos afirmar que al tener en cuenta la visión de los estudiantes se propende a una mejora en las prácticas educativas desde la motivación que les brinda la participación activa (Aparicio \& Ostos, 2018). Así mismo, el cambio en la metodología de enseñanza-aprendizaje concentra las acciones del profesor en los intereses de los estudiantes, por tanto, las orientaciones didácticas reforzarán los procesos de investigación escolar en la enseñanza escolar.

\section{Referencias bibliográficas}

Aparicio, O.Y., \& Ostos, O.L. (2018). Las TIC como herramientas cognitivas para la investigación. Revista Interamericana de Investigación, Educación y Pedagogía, RIIEP, 11(1). https://orcid.org/0000-0003-3535-6288. https://orcid.org/0000-0002-6477-9872

Area, M. y Adell, J. (2009). E-learning: Enseñar y aprender en espacios virtuales. En De Pablos, J. Tecnología educativa. La formación del profesorado en la era de Internet (pp. 391-424). Málaga: Aljibe.

Bennis, W. y Biederman, P. (1997). Organizing genius: The secrets of creative collaboration. Toronto: Addison Wesley.

Brooks, F. B. y Donato, R. (1994). Vygotskyan approaches to understanding foreign language learner discourse during comunicatives tasks. Hispania, 77(1).

Chambers, A. (1985). Booktalk Occasional writing on literature and children. London: The Bodley Head.

de la Calle, C. V., Malaver, M. O., Gallego, J. D. M., Rodríguez, M., Flórez, J. C., Henao, C. E. \& Saldaña, R. (2014). Aportes de los doctorados de educación en ciencia, tecnología y sociedad, desde la sistematización de sus investigaciones doctorales científicas y formativas, 2000-2010. Revista Interamericana de Investigación, Educación y Pedagogía, RIIEP, 7(1). DOI: https://doi.org/10.15332/s1657-107X.2014.0001.04

Durban, G. y Blasco, A. (2012). Model Tres Fases 3•3•3. Recuperado de: https://sites. google.com/a/xtec.cat/cinfo-aula/model-2-fases

Eduteka. (2007). Modelo Gavilán 2.0. una propuesta para el desarrollo de la Competencia para Manejar Información (CMI). Recuperado de: http://www.eduteka. org/pdfdir/ModeloGavilan.pdf

Ferreyra, H. A. (2014). Mesas Socioeducativas para la Inclusión y la Igualdad. Un programa "De todos con todos". Una experiencia en construcción. Revista Interamericana de Investigación, Educación y Pedagogía, RIIEP, 7(2). DOI: https://doi. org/10.15332/s1657-107X.2014.0002.01 
Gardner, H. (1983). Frames of Mind: The Theory of Multiple Intelligences. New York: Basic Books.

Gordon W. (2003). Acción, conversación y texto. Aprendizaje y enseñanza a través de la investigación. Sevilla. Publicaciones M.C.E.P.

Hong, N., Jonassen, D. y McGee, S. (2003). Predictors of well-structured and illstructured problem solving in an astronomy simulation. Journal of Research in Science Teaching, 40(1), 6-33.

Jonassen, D. H. (1997). Instructional design model for well-structured and illstructured problem-solving learning outcomes. Educational Technology: Research and Development, 45(1), 65-95.

Konieczny, P. (2015). Lorenzo García Aretio: bases, mediaciones y futuro de la educación a distancia en la sociedad digital. Revista Interamericana de Investigación, Educación y Pedagogía, RIIEP, 8(1). DOI: https://doi.org/10.15332/s1657107X.2015.0001.08

Langer, E. (2016). La construcción de confianza para el estudio de prácticas de resistencia en la escolarización de jóvenes en contextos de pobreza urbana. Revista Interamericana de Investigación, Educación y Pedagogía, RIIEP, 9(2). DOI: https://doi. org/10.22490/25391887.1945

Lau, Jesús. (2007). Directrices sobre desarrollo de habilidades informativas para el aprendizaje permanente. Boca del Río, Veracruz, México: IFLA. Recuperado de: http:// www.ifla.org/files/assets/information-literacy/ publications/ifla-guidelines-es.pdf

Lissón, A. (1989). Estudi o Investigació. Barcelona: Escola Costa i Llobera (documento fotocopiado).

Monereo C. y Fuentes M. (2008). La enseñanza y el aprendizaje de estrategias de búsqueda y selección de la información en los entornos virtuales. En Coll, C. y Monereo, C. (Eds.) Psicología de la educación virtual. Aprender y enseñar con las Tecnologías de la Información y la Comunicación (pp. 368 - 408). Madrid: Morata.

Newman, J. (1987). Learning to teach by uncovering our assumptions. Language Arts, 64(7), 727-737.

Onrubia, J., Colomina, R. y Engel, A. (2008). Los entornos virtuales de aprendizaje basados en el trabajo en grupo y el aprendizaje colaborativo. En Coll, C. y Monereo, C. (Eds.) Psicología de la educación virtual. Aprender y enseñar con las Tecnologías de la Información y la Comunicación (pp. 233 - 252). Madrid: Morata.

Papert, S. (1982). Desafío a la mente: Computadoras y educación. Buenos Aires: Galápago. 
Pérez, T. H. P. (2013). Aproximaciones al estado de la cuestión de la investigación en educación y derechos humanos. Revista Interamericana de Investigación, Educación y Pedagogía, RIIEP, 6(1). DOI: https://doi.org/10.15332/s1657-107X.2013.0001.05

Pérez, T. H. (2014). Colombia: de la educación en emergencia hacia una educación para el posconflicto y la paz. Revista Interamericana de Investigación, Educación y Pedagogía, RIIEP, 7(2). DOI: https://doi.org/10.15332/s1657-107X.2014.0002.06

Piaget, J. (1980). Psicología y pedagogía. Barcelona: Ariel.

Popper, K. (1957). El objetivo de la ciencia. En Miller, D. (Ed.). Popper: escritos selectos. México: Fondo de Cultura Económica.

Pozuelos, F. y Travé, G. (2005) Aprender investigando, investigar para aprender. El punto de vista de los futuros docentes. Una investigación en el marco de la formación inicial de magisterio y Psicopedagogía. Investigación en la Escuela, 54, 2-25.

Punt de Lectura. (2014). Competència informacional. [Mensaje en blog]. Recuperado de : https://drive.google.com/file/d/0B8C82K7A5FOqNDI3MkZPdUFHS1E/edit

Quintana, J. (2014a). 7 passos per fer una tasca amb informació: una proposta oberta. Recuperado de: http://www.ub.edu/ntae/jquintana/7passos7_2014.pdf

Quintana, J. (2014b). Recull d'alguns models rellevants per al treball amb informació. Recuperado de: http://www.ub.edu/ntae/jquintana/ModelsCMI.pdf

Rosebery, A., Warren B. y Conant, F. (1992). Appropriating scientific discourse: Findings from languages minority classrooms. Journal of the Learning Sciences, 2(1), 61-94.

Short, K. y Burke, C. (1991). Creating curriculum: Teachers and students as a community of learners. Portsmouth, NH: Heinemann.

Tahull, J. (2016). Modernidad, educación y género. El proyecto inacabado. Revista Interamericana de Investigación, Educación y Pedagogía, RIIEP, 9(2), 159-178. DOI: https://doi.org/10.22490/25391887.1947

Tonucci, F. (1982). Metodología de la investigación en el aula. Boletín de Acción Educativa, (39), 15-20.

Van Tassell, M. A. y Gianotti, M. A. (1994). Moving between worlds: Talk during writing Workshop. En Wells, G., et al., Changing schools from within: Creating communities of inquirí (pp. 3759). Toronto: OISE Press.

Viejo, C. M., Cabezas, I. L., \& Martínez, M. D. J. I. (2013). Las redes de académicas en la docencia universitaria. Revista Interamericana de Investigación, Educación y Pedagogía, RIIEP, 6(2). DOI: https://doi.org/10.15332/s1657-107X.2013.0002.03 
Vygotsky, L. S. (1987): Thinking and speech. En Rieber, R. W. y Carton, A. S. (Eds.). The collected Works of L. S. Vygotsky, Volume 1: Problems of general psychology (pp. 39285). New York: Plenum.

Wells, G. (1997). From guessing to predicting: Progressive discourse in the learning and teaching of science. En Coll, C., Edwards, D. (Eds.). Teaching learning and classroom discourse: Approaches to the study of educational discourse (pp. 67-87). Madrid: Fundación Infancia y Aprendizaje.

Wells, G. (1999). Dialogic inquirí: Towards a sociocultural practice and theory of education. New York: Cambridge University Press. 